\title{
Féeries
}

Études sur le conte merveilleux, XVII ${ }^{\mathrm{e}} \mathrm{XIX} \mathrm{X}^{\mathrm{e}}$ siècle

$12 \mid 2015$

À la croisée des genres

\section{Banville au pays des merveilles : idéal et illusion}

Banville in Wonderland: Ideal and Illusion

Jean-Louis Cabanès

\section{(2) OpenEdition}

Journals

Édition électronique

URL : http://journals.openedition.org/feeries/966

ISSN : 1957-7753

Éditeur

UGA Éditions/Université Grenoble Alpes

Édition imprimée

Date de publication : 15 octobre 2015

Pagination : 79-92

ISBN : 978-2-84310-306-3

ISSN : 1766-2842

Référence électronique

Jean-Louis Cabanès, « Banville au pays des merveilles : idéal et illusion », Féeries [En ligne], 12 | 2015,

mis en ligne le 15 octobre 2016, consulté le 08 septembre 2020. URL : http://journals.openedition.org/ feeries/966

\section{(c) Féeries}




\author{
Jean-Louis Cabanès \\ Université Paris Ouest Nanterre La Défense / CSLF (EA 1586)
}

\title{
BANVILLE AU PAYS DES MERVEILLES : IDÉAL ET ILLUSION
}

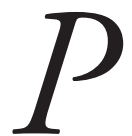

OĖTE FUNAMBULESQUE, Banville ne cesse de faire surgir des fées au sein de ses œuvres, qu'il se souvienne des contes de Perrault ou de ceux de Madame d'Aulnoy, qu'il se réfere aux diverses formes de féeries (pantomimes magiques, féeries-vaudevilles, mélodrames-féeries) données sur les scènes parisiennes au XIX ${ }^{\mathrm{e}}$ siècle. En I855, il publie une longue nouvelle, La Vie d'une comédienne, centrée sur un personnage lisant des contes de fées et jouant dans des féeries puis, en I882, des Contes féeriques modernes. En 1883 , il fait représenter une féerie, Riquet à la houppe, qui sera suivie, en I887, du Baiser. Naissant de livres déjà existants, dénotant parfois, à l'inverse, la possibilité de créer un univers informé par la subjectivité esthétique d'un créateur original, le merveilleux cristallise une axiologie, elle-même subordonnée à la multiplication des allégories. Associé au rêve, parfois auréolé de références religieuses, ouvrant même sur une esthétique du ravissement, il se déploie entre parodie sérieuse et second degré joueur, appelant à l'occasion un sourire tout prêt à se métamorphoser en ironie. Il est moins une tonalité, une modalité des fictions, que le révélateur des grands écarts banvilliens. D'une part, l'auteur des Odes funambulesques semble souscrire au "principe féerique", défini par Louis Bouilhet dans une lettre à Flaubert du 6 octobre i863: "Bien établir, comme une religion indubitable, que nous sommes entourés d'êtres invisibles, plus forts que nous, lesquels êtres se manifestent quand bon leur semble et dans les siècles qui leur conviennent ${ }^{\mathrm{I}}$. " Les deux féeries écrites par Banville, Riquet à la houppe et Le Baiser, sont fondées, en effet, sur ce "principe». Dans ses Contes féeriques, l'écrivain, comme il le dit lui-même, «mêle» les fées «à ce peuple infini / Que nous ont peint Balzac

I. G. Flaubert, Correspondance, édition établie, présentée et annotée par J. Bruneau, Paris, Gallimard, coll. «Bibliothèque de la Pléiade», I991, t. III, p. 959. 
et Gavarni ${ }^{2}$ ». "Mêler» suggère la coexistence pacifique de deux mondes distincts : le merveilleux s'insère tout naturellement dans l'univers réel. D'autre part, dans La Vie d'une comédienne, Minette, lectrice naïve de contes de fées qu'elle croit fidèlement mimétiques, subit les violences de ses parents qui l'exploitent, qui la battent et s'entretuent. La victimisation du personnage impose dans cette nouvelle une noirceur qui est tout à l'opposé des contes dont Minette s'enchante. La référence féerique se modalise ainsi différemment selon les genres, elle devient particulièrement complexe lorsque, dans un cadre générique donné (nouvelle, conte), la diégèse inclut des scènes se rapportant à d'autres genres, notamment aux féeries théâtrales. Étudier les modes d'apparition des fées ou des puissances surnaturelles ou, à l'inverse, leur effacement, tenter de saisir les migrations génériques du merveilleux revient certes à évoquer des questions d'ordre rhétorique et esthétique, mais aussi à se demander ce qui subsiste de la magie des fables lorsqu'une allégorie fait office de moralité explicite, ou lorsque le conte bleu bute sur le pathos d'un réalisme noir.

Le merveilleux dans les Contes féeriques ou dans les féeries écrites par Banville n'est pas apparenté à l'insolite, à l'incongru, il ne résulte pas d'un regard attentif aux spectacles étonnants offerts par la ville moderne. Il ne naît pas d'une illumination, d'une rencontre, il ne ressortit pas davantage à une esthétique de la trouvaille, liée au bizarre, à la surprise, à l'irruption d'un inattendu. En revanche, il participe parfois d'un second degré, d'une réécriture que les titres indiquent explicitement: Riquet à la houppe, Riquette à la houppe, et fait surgir des figures connues : la fée Urgèle, le prince charmant, Gracieuse et Percinet, la méchante marâtre. L'écrivain, dans ses Contes féeriques, ne cesse, en outre, de renvoyer à des objets magiques : baguettes, bagues, talismans. La scène topique de la métamorphose d'une vieille femme en une belle fée survient de manière récurrente. Étienne Silvant sauve une chiffonnière qui manque d'être écrasée par des chevaux emballés. Transfigurée, se présentant comme la fée Eryx, elle offre un cigare magique à son sauveur ${ }^{3}$. Paule de Trénil, qui s'est montrée charitable à l'égard d'une pauvresse, voit cette dernière devenir la fée Néis 4 . Riquette à la houppe, tout aussi charitable, donne un verre de coco glacé et un éventail à une vieillarde accablée par la chaleur; celle-ci se métamorphose et dévoile son identité : elle est la fée Taras'. Le merveilleux, dans

2. Th. de Banville, «Ballade pour célébrer les fées parisiennes», Contes féeriques, Paris, Charpentier, I882, p. VII.

3. Voir «La Chiffonnière», ibid.

4. Voir «Le dernier Bal», ibid.

5. Voir «Riquette à la houppe», ibid. 
les Contes féeriques, repose ainsi sur des ressorts traditionnels que Banville s'amuse à faire jouer dans un contexte moderne.

Le décor des apparitions est précisément délimité : rue Saint-Merri, rue Maubuée ${ }^{6}$, théâtre des marionnettes du jardin du Luxembourg 7 , bal de l'Opéra ${ }^{8}$, tandis que le nom des fées qui s'imposent à la vue des personnages renvoie parfois à la Grèce antique : Tyro, Eryx, Néis. Le merveilleux assure ainsi la rencontre de deux temporalités distinctes, ce qui n'induit pas pour autant une dissonance, encore moins une inquiétante étrangeté. Certes l'assimilation des fées à des figures empruntées à la mythologie grecque produit une tension polémique entre l'éloge de l'Antiquité que leur intrusion bienveillante présuppose et la mise en relief des manques, de la cruauté d'un monde contemporain soumis au règne de l'argent et insensible à la qualité esthétique des œuvres d'art. Mais la venue des fées n'induit aucun trouble, aucun émoi chez ceux qui les voient apparaître. C'est plutôt la naturalité du merveilleux que l'écrivain entend souligner dans ses contes, en situant les apparitions dans le cadre de la vie ordinaire. Une petite fille, Georgette, persécutée par sa marâtre, voit une fée surgir de sa boîte à ouvrage9. Pierre Alek, un jeune collégien, découvre la fée Myr sous des feuilles de marronnier ${ }^{10}$. Il l'installe dans son pupitre. On constate surtout que les Contes féeriques multiplient les références à la vie parisienne, font vivre un personnel que l'on retrouve souvent dans Le Gaulois ou dans Gil Blas : écrivains, artistes, acteurs, mondains. Satan en personne, mécontent des traits que les romantiques lui ont prêtés, vient se montrer au bal de l'Opéra : il veut être dessiné par Gavarni afin d'être représenté comme un diable moderne ${ }^{\text {II }}$. Parfois, sous une forme allusive, l'écrivain renvoie à l'actualité littéraire. Un directeur de théâtre espère «avoir un drame expérimental et une féerie scientifique, où tout se dénoue par la géographie ${ }^{12} »$. Banville articule ainsi, avec humour, deux conventions, celle qui régit le conte merveilleux, celle qui gouverne la chronique de la vie parisienne ${ }^{13}$.

\footnotetext{
6. Voir «La Chiffonnière», ibid.

7. Voir «Les Huit sous de Pierrot», ibid.

8. Voir «Bal à l'Opéra», ibid.

9. Voir «Georgette et Zozo», ibid.

Io. Voir «Un Meissonier», ibid.

II. Voir «Bal à l'Opéra", ibid.

I2. «La Figurante», ibid., p. 6.

I3. Il nous apparaît significatif que le titre donné à un conte soit précisément «Chronique parisienne».
} 
Si ce clichage généralisé donne au lecteur le sentiment d'être en pays de connaissance, la naturalité des fées est surtout la conséquence d'un effet optique, d'une illusion produite par un conteur qui feint d'accréditer "le principe de féerie", pour rendre le surnaturel naturel dans des contes dont certains "réfléchissent» le merveilleux par des jeux métatextuels. Cette réflexivité est tout particulièrement à l'œuvre lorsque les fées prennent plaisir à "montrer leur forme» dans le cadre d'un théâtre où l'on joue des féeries. Leur présence en un lieu que l'on dira "exposé» invite à s'interroger sur l'idée de représentation et sur la portée ambivalente de la parodie chez Banville. On est même conduit à se demander si l'allégorie, dès lors qu'elle se trouve subordonnée à l'existence fictionnelle d'un personnage agissant, support figural et actantiel d'une idée, ne participe pas à ce dédoublement, à ce fonctionnement par paire que Banville semble tout particulièrement affectionner.

Qui voit les fées? Zameith, qui joue le rôle de Jocrisse dans une féerie "contempl[e] avec fixité [...] une quatrième fée qui, placée près des trois autres n'[est] soutenue par rien, et para[ît] voltiger réellement comme un oiseau $[\ldots]^{14}$ ». C'est la donnée initiale de «La Figurante». Dans «Les Huit sous de Pierrot", une jolie petite fée déguisée en marionnette est certes suspendue à des fils, mais le narrateur aperçoit ses ailes, elle n'est pas «de bois» mais de chair ${ }^{15}$. Si les spectateurs, qui forment le public du théâtre, ne différencient pas la semblance de la présence réelle, en revanche le bouffon-poète qui, dans "La Figurante», s'est pris de passion pour une fée, et l'écrivain-narrateur des "Huit sous de Pierrot» ne commettent pas semblable méprise. Dans le premier conte, le directeur de théâtre, Jaquerod, "Parisien sceptique», funambule involontaire («il dansait sur la corde roide des expédients [...], tantôt sans balancier, tantôt avec les premiers balanciers venus $\left.{ }^{16} »\right)$, sait, lui aussi, repérer la vraie fée au milieu de ses fausses consœurs. Bien qu'il laisse "ses idées à la porte du théâtre comme on doit laisser tout espérance à la porte des enfers ${ }^{17}$ ", il se voit accorder une voyance comparable à celle du Jocrisse, parce qu'il aime Shakespeare dont il lit les œuvres dans le secret de son appartement. Voir les fées est ainsi un don réservé aux artistes, à ceux qui pourraient l'être, ou bien encore aux enfants (Georgette, Pierre Alek).

\footnotetext{
I4. «La Figurante», ibid., p. 2.

15. "Les Huit Sous de Pierrot», ibid., p. I8I.

16. "La Figurante», ibid., p. 3.

I7. Ibid., p. 4.
} 
Si l'on déplace le regard du côté des êtres merveilleux, on constate que leur incarnation sensible sur la scène du monde, et bien évidemment sur la scène du conte féerique moderne, est subordonnée à la reconnaissance qu'on leur accorde. Pour que les fées prennent forme il importe que l'on fasse confiance à la vertu poétique du merveilleux. Dans un monde désenchanté par la science et par le règne de l'argent, elles ne peuvent se montrer que sur la scène des théâtres où l'on joue des féeries. Le faux, en quelque sorte, les protège. C'est ce qu'affirme, dans «La Figurante», la fée Tyro. Le merveilleux, dans ce conte, se présente donc sur deux modes, l'un factice, «travesti», l'autre que l'on dira "originel». Tyro ne se «mêle» pas au «peuple infini » représenté par Balzac ou par Gavarni ${ }^{18}$, mais elle participe à une représentation que sa présence dénonce implicitement comme telle. On comprend la méfiance du directeur de théâtre. Celui-ci redoute qu'une fée authentique soit en dysharmonie avec l'artifice qui règne sur un théâtre marqué par des "clous» spectaculaires.

Le jeu avec les frontières du faux et du vrai se concrétise tout particulièrement dans le finale du conte. Trois actrices qui tiennent le rôle de fées s'amusent à faire semblant d'accorder à Adolphe, le fils du directeur, dont on fête la naissance, trois dons "pour de faux", celui d'être malin et rusé, celui de plaire aux femmes, celui d'avoir beaucoup d'argent. On mime une performativité, signe du merveilleux, et on en rit. Survient la fée Tyro qui octroie à l'enfant un don véritable : "Cher petit [...], tu seras poète!» "Je suis déshonorér ${ }^{19}$ !", répond Jaquerod. Banville s'amuse, dans "La Figurante», à complexifier la référence féerique en jouant une petite musique à deux temps. Il dispose, en effet, les personnages et l'instance narrative en couples successifs et symétriques : Tyro / les trois actrices (la vraie fée et les fausses fées), Zameith /le directeur du thêâtre (tous deux savent reconnaître en Tyro une fée, mais leurs sentiments s'opposent), le narrateur du conte / les spectateurs de la féerie (le premier est omniscient, les seconds sont aveugles). Cette binarité généralisée permet d'exploiter la polysémie essentielle à l'idée de représentation en rapportant cette dernière à la parodie, au faux-semblant, au mensonge théâtral opposé à la «vérité féerique $^{20}$ ». On peut lire, en effet, "La Figurante» comme une parodie sérieuse de conte merveilleux qui inclut, dans son finale, une séquence de parodie blagueuse, que corrige un don véritable, suscitant à son tour une ironie à entrées multiples. Le "Je suis déshonoré» de Jacquerod se présente

I8. Voir supra, "Ballade pour célébrer les fées parisiennes», Contes féeriques, p. VII.

19. "La Figurante», ibid., p. 9.

2o. Ibid., p. 6. 
comme un bon mot, une pointe, un trait d'esprit, une moralité ironique. Pour le directeur de théâtre, la belle fée Tyro tient d'une Carabosse. Le merveilleux a droit d'existence dans les livres, il sollicite l'imagination du lecteur, ses capacités poétiques, mais son intrusion dans la réalité, même si la fée sauve Jaquerod d'une éventuelle faillite en faisant revenir, comme dans la pièce de Balzac, Le Faiseur, un Godeau que l'on n'attendait plus, s'apparente à une ironique malédiction. Devenir poète dans une période où le lyrisme a perdu toute auréole, n'est-ce pas exercer le plus incongru des métiers, comme le montre une nouvelle de Banville, "Le Festin des Titans", dans laquelle, lors d'un étrange concours, un jeune homme, poète lyrique vivant de son état, remporte, haut la main, le prix de la profession la plus excentrique ${ }^{21}$ ?

À considérer l'ensemble des Contes féeriques, on constate que la parodie est le plus souvent dénoncée comme une blague désacralisante. Dans «Un vieux vaudeville», Farette, poète manqué, après avoir écrit Les Folies de Sparte, envisage de faire représenter La Jarretière d'Eurydice. Il sera puni par les Dieux qu'il ridiculisait dans ses vaudevilles. Muet, les mains paralysées, il meurt sur la scène de la Gaîté, où il joue le rôle de figurant, pendant un entracte d'Orphée aux enfers. La reproduction fidèlement mimétique ne vaut pas mieux que le travestissement vaudevillesque. Dans «Parfait amour», Anatole Passeron, projection d'Henry Monnier, dessine des bourgeois chauves, ventrus, obèses. Il les met en scène et finit par les jouer si bien qu'il se «trouv[e] parfaitement identique à ceux qu'il voulait imiter ${ }^{22}$ ». L'objet magique, dans ce conte féerique, est un flacon de verre, rempli d'une liqueur appelée "parfait amour», et "moulé de façon à représenter le buste de $\mathrm{M}$. Thiers ${ }^{23}$ ». À peine l'artiste a-t-il acheté cette extraordinaire merveille mimétique, à peine a-t-il consommé quelques gorgées de "parfait amour» qu'il ne peut plus faire autre chose qu'imiter, au point de susciter une fantasmagorie du même. Le bourgeois adhère à son enveloppe, il est d'autant plus plein de lui-même qu'il est creux, mais le comédien-dessinateur ne lui donne vie et forme qu'en adoptant sa posture et son langage, au risque de perdre son identité. Le parfait amour est fusionnel. Le typique M. Thiers, abrégé allégorique d'une classe sociale, en lançant une malédiction grotesque, a transformé en bourgeois celui qui mimait la bourgeoisie. Les contes de Banville instaurent ainsi une hié-

2I. Cette nouvelle, d'abord parue dans la presse en 1848 (Le Corsaire, 24 et 25 novembre) s'adjoindra en I855 à l'édition de La Vie d'une comédienne, chez Michel Lévy.

22. Th. de Banville, "Parfait Amour», Contes féeriques, ouvr. cité, p. 256.

23. Ibid., p. 253. 
rarchie de valeurs. Au plus bas de l'échelle, véritable degré zéro de l'esthétique, s'impose un réalisme qui accomplit ce miracle de confondre l'icône et l'objet représenté; vient ensuite le travestissement burlesque ou vaudevillesque presque aussi haïssable; la féerie spectaculaire vaut mieux, mais elle fait miroiter une poésie du toc. L'idéal serait d'atteindre la "vérité féerique» ou de la susciter, mais peut-on éviter la parodie? Si cette question se pose à propos des Contes féeriques modernes, on peut aussi la soulever à propos des féeries théâtrales. Il est significatif que dans Le Baiser, où une vieille femme se transforme en fée Urgèle, Pierrot déclare son amour à cette dernière en se souvenant de Tartuffe: «Mon innocence commence à me peser / Et pour être Pierrot, je n'en suis pas moins homme ${ }^{24}$.» Les contes, les féeries écrits par Banville, parce qu'ils sollicitent la connivence du lecteur ou du spectateur, parce qu'ils se veulent humoristiques et allusifs, ressortissent toujours au second degré ou le suscitent ponctuellement. Peut-on se défaire de l'empois du déjà dit, lorsqu'on veut, au XIX ${ }^{e}$ siècle, écrire des contes merveilleux modernes? N'est-on pas condamné à l'ironie, qui implique l'emploi en mention d'un mot ou d'un motif?

On reformulera cette question en substituant au "qui voit les fées» l'interrogation suivante : "qui les fait naître"? C'est ouvrir une autre scène, celle de l'intériorité. Dans "Le Renégat», Claude Justel laisse s’emplir la "chambre noire de son cerveau ${ }^{25}$ " d'images, de rêves, en partie alimentés par ses lectures. Il se met à dialoguer «avec une invisible compagne ${ }^{26}$ » qui s'impose d'abord comme une voix, qui devient ensuite une vision, puis une forme pleinement perceptible. De la solitude de Claude Justel, de ses lectures, a donc surgi la fée Euryale dont ce jeune poète reconnaît les traits parce qu'ils sont tels qu'il se les était figurés. En se souvenant de Gautier, on pourrait dire que Claude Justel "port[e] en lui le microcosme, c'està-dire un petit monde complet d'où il tire sa pensée et la forme de ses œuvres». "Les artistes qui ont le microcosme, ajoutait Gautier, lorsqu'ils veulent produire, regardent en eux-mêmes et non au-dehors ${ }^{27}$." Euryale est à la fois une figure surgie du microcosme intérieur et l'allégorie de l'imagination créatrice.

Si le mystique doit prendre garde aux promesses du monde, il en est de même pour le poète lyrique. Le démon tentateur, pour Claude Justel,

24. Th. de Banville, Le Baiser, Théatre complet, P. J. Edwards et P. S. Hambly (éds), Paris, Honoré Champion, t. III, 20I2, p. 245.

25. Th. de Banville, «Le Renégat», Contes féeriques, ouvr. cité, p. 200.

26. Ibid., p. 20 .

27. Th. Gautier, «Salon de I839", La Presse, 4 avril I839. 
c'est le directeur de la Revue Continentale, Ulric Lagès, qui lui suggère d'écrire des poèmes médiocres en imitant ceux d'Eugène Bertina. On aura reconnu, dans la Revue Continentale, la Revue des Deux Mondes et Bertina renvoie à Louis-François Bertin, plus vraisemblablement à son fils, Édouard Bertin, qui furent tous deux directeurs du Journal des Débats. Justel connaît le succès, se marie richement (comme Pierre Grassou), il est enfin élu à l'Académie. Le jour de sa réception, il voit le cortège funèbre de la fée Euryale se multiplier sur «les feuilles vertes des habits des académiciens ${ }^{28}$ ». Il tombe évanoui. Une fois encore la parodie, ce que Banville appelle "le travail de singe ${ }^{29}$ ", s'impose comme le tombeau du merveilleux. Mais ce n'est plus le travestissement vaudevillesque qui est visé. Le conte tient d'une charge contre l'académisme et le pompiérisme que Claude Justel s'est amusé à imiter en écrivant de "pompeuses niaiseries ${ }^{30}$ ", au risque de se laisser fasciner par elles. On a changé d'optique, de lunette d'approche. Il ne s'agit plus d'opposer la vérité féerique à la blague des féeries-vaudevilles, mais de souligner que la création originale présuppose une écoute intérieure, une confiance dans l'œil de l'esprit, susceptible non plus seulement de voir les fées déjà existantes mais de faire apparaître des fées nouvelles, de susciter de l'innommé. Pour autant, l'originalité demeure un horizon fuyant et l'on a vu que Banville, dans "Le Renégat", multiplie les clés, s'amuse avec le second degré. D’un côté, les Contes féeriques ne cessent de postuler l'existence des fées, de les naturaliser, de les mêler à la vie contemporaine et l'écrivain, en actualisant ce postulat dans la trame narrative, trouve matière à enchanter occasionnellement le récit en évoquant, comme dans "Un amateur», au gré d'amples descriptions, un festin somptueux censé combler les rêveries sensuelles de Paul Cingal. De l'autre côté, et l'on serait tenté de dire dans la description somptueuse, saturée de qualifiants de cette fête à l'antique, presque gautiériste, les Contes féeriques ne cessent d'emprunter une matière déjà existante. L'invention banvillienne se manifeste avant tout par des jeux métatextuels, par les reflets miroitants que l'écrivain sait organiser en combinant sourire et polémique, par les arabesques du récit qui enlacent le merveilleux traditionnel dans les volutes d'une intrigue en partie allégorique, par l'alliance de l'ironie et de la désillusion. La fantaisie qui caractérise les Contes féeriques produit une sorte de battement, de voltige sémantique

28. Th. de Banville, «Le Renégat», Contes féeriques, ouvr. cité, p. 207.

29. Ibid., p. 205.

30. Ibid., p. 206. Notons que l'un des contes s'appelle «Le Pompier». Il a pour héros, si l'on peut dire, un peintre qui emprunte beaucoup à Ingres. 
qui ne peut jamais s'arrêter, s'immobiliser, car le merveilleux tient d'un originel qui appelle l'écrivain à devenir original. Entre les deux pôles, les Contes féeriques balancent avec humour. Le merveilleux ne se fixe que pour donner aux fées une valeur discriminante, celles-ci surgissent, s'imposent, disparaissent, s'envolent, s'effacent en instruisant indirectement le procès d'un monde apoétique. Le narrateur des Contes les crédite d'une existence pleine et entière, leur accorde une fonction dans l'intrigue pour mieux suggérer une perte. Personnages essentiels de ces petites fictions qui oscillent entre parodie sérieuse et dénonciation de la parodie, elles s'enlèvent sur un fond négatif.

Ces remarques pourraient en partie s'appliquer à la nouvelle publiée en I855, La Vie d'une comédienne, qui s'apparente à une fable dont la fin tient à la fois d'une moralité exemplaire et d'une allégorie. Si on peut voir dans ce récit la matrice des Contes féeriques, le genre de la nouvelle avait contraint Banville, en I855, à envisager le merveilleux tout autrement que dans des textes publiés en recueil vingt-sept ans plus tard. Aucune fée réelle ne hante un récit dans lequel les Contes de Madame d'Aulnoy jouent un rôle pourtant essentiel. On n'y rencontre aucune parodie, même si la question de la semblance, de la représentation, y est centrale. Exalter la simplicité de l'héroïne, Minette, c’est nécessairement bannir le second degré.

La Vie d'une comédienne a pour cadre principal le théâtre de la Gaieté. Il s'agit, précise le narrateur, de l'un de ces théâtres populaires où l'on divertit l'ouvrier des faubourgs en lui faisant oublier «ses poignantes misères de chaque jour" par «les fictions où le merveilleux abond[e] comme dans les contes de fées et les récits des Mille et une Nuits ${ }^{31} »$. Minette a pour parents deux saltimbanques, Adolphina et "le Capitaine», qui la battent et qui se battent, qui s'enivrent et qui l'exploitent. Un tournant est pris lorsqu'elle apprend à lire dans les Contes de Madame d'Aulnoy. Si tout enfant, elle se délivrait de son existence sordide par les rêves, désormais «elle p[eut] vivre en idée loin de la hideuse réalité qui la tu[e] $]^{32}$. Elle console ainsi sa souffrance par des visions extatiques qui lui permettent, par le truchement de la lecture, de s'imaginer heureuse. Ses parents, qui l'accablent de tâches, l'introduisent au théâtre de la Gaieté; on y représente Gracieuse et Percinet, féerie qu'elle vit comme si c'était une histoire vraie. Elle tombe amoureuse de l'acteur Couturier qui joue le prince charmant et qu'elle identifie à ce rôle. En contrepoint, le couple parental s'enfonce dans le

31. Th. de Banville, La Vie d'une comédienne, ouvr. cité, p. I-2.

32. Ibid., p. 20. 
crime. Le Capitaine tue Adolphina, qui s'était éprise d'un jeune homme; le meurtre accompli, il s'élance par la fenêtre et se brise les jambes. Minette, qui a assisté à la scène, sombre dans le délire. Conduite à l'hôpital, elle est initiée à la religion. Comme elle ne fait pas le partage entre le réel et le surnaturel, elle ne comprend ni la mort, ni la vie future, et «appliqu[e] à notre vie terrestre toutes les diverses espérances de résurrection et d'existence purifiée qui nous donnent la force de supporter tous les maux ${ }^{33}$ ». La jeune fille, en la réduisant à du merveilleux, prive ainsi la religion de transcendance. Les représentations idéales que l'imagination, la lecture des contes de fées ou celle des livres saints font naître doivent s'accomplir ici-bas. Le théâtre est conçu par Minette comme une étape, il est une antichambre transitoire où la chrysalide de l'idéal est sur le point d'accomplir sa métamorphose. Les croyances naïves de Minette vont lui coûter cher. Couturier fréquente les prostituées. À 27 ans, il est chauve. Voyons-y le coup de pied de Vénus, ou si l'on préfère la trace d'une probable syphilis. Pour que le tableau soit complet, ce comédien s'éprend d'une actrice, la Bambinelli. Minette, qui a reconnu en sa rivale «la menaçante beauté qui avait désolé ses rêves ${ }^{34}$ ", se laisse tomber du haut du fil qui la soutenait et qui lui permettait de jouer «l'ange». La chute de Minette est symétrique du saut prodigieux du saltimbanque qui, dans les Odes funambulesques, va «rouler dans les étoiles ${ }^{35}$ ».

Jean-Baptiste Montaubin ${ }^{36}$, Jean-François les Bas-bleus ${ }^{37}$, héros éponymes de deux contes de Nodier, avaient eux aussi des visions, ils semblaient aux yeux du monde l'incarnation de ce que Banville appelle, à propos de Minette, "une douce et poétique folie ${ }^{38}$ ». Mais les transports extatiques de ces âmes simples les mettaient en contact avec une réalité supérieure. En revanche, les représentations poétiques que Minette se donne à elle-même, les féeries dans lesquelles elle joue, les contes de fées qui nourrissent ses rêves ne peuvent être rapportés à une quelconque surnature, ils ne présupposent pas une articulation poétique de l'imagination créatrice et de l'ontologie. En I852, George Sand, dans sa préface à la réé-

33. Ibid., p. 73 .

34. Ibid., p. 93.

35. Th. de Banville, «Le saut du tremplin», Odes funambulesques, Alençon, Poulet-Malassis et de Broise, 1857 , p. 240.

36. Ch. Nodier, "Baptiste Montauban ou l'Idiot», Le Conteur, recueil de tous les temps et de tous les pays, Paris, Charpentier, I833, repris dans les Contes de Charles Nodier, Paris Charpentier, I840.

37. "Jean-François les Bas-bleus", Les Cent-et-une Nouvelles des Cent-et-un, Paris, Ladvocat, I833, t. I, repris dans les Contes de Charles Nodier.

38. Th. de Banville, La Vie d'une comédienne, ouvr. cité, p. 38. 
dition de Jeanne $e^{39}$, présente son héroïne comme «une vierge de Holbein », comme "une femme primitive», "une Jeanne d'Arc ${ }^{40}$ ». Elle croit dans la puissance magique des fées et sa religion naïve tient d'un syncrétisme : "La Vierge Marie et la grand' fade se confondaient étrangement dans l'imagination poétiquement sauvage de la bergère d'Ep-Nell ${ }^{4 \mathrm{r}}$." Si la jeune comédienne de Banville est à la fois sublime et simple, comme l'était Jeanne, sa croyance dans la réalité du merveilleux tient d'une erreur herméneutique qui ne lui permet de comprendre ni ce qui est représentation, ni ce qui est symbole. Elle ne sait pas faire le partage entre les féeries dans lesquelles elle joue et la réalité, entre l'histoire sainte qu'une bonne sœur lui fait découvrir et l'univers des contes. Ni le jeu théâtral, ni une lithographie — celle de la Fée des Eaux que Madame Paul donne à Minette —, ne sont considérés pour ce qu'ils sont. Dans l'univers de la comédienne-enfant, parce qu'elle est simple, font défaut le mensonge, l'opposition de l'apparence et de l'être. La plénitude merveilleuse des contes de fées conduit ainsi Minette à une négation totale des signes comme représentants analogiques, indiciaires, iconiques d'une réalité absente qu'ils auraient pour mission de représenter ou de signifier. Parce que Minette conjoint la simplicité, la sublimité et l'erreur, elle semble proche de la Félicité de Flaubert. Dans le paradigme des cours simples, elle figure un personnage transitif qui nous conduit de Nodier à Flaubert, en passant par Sand.

Si Banville élabore, dans cette nouvelle, un romanesque noir du défaut herméneutique, Minette, transfigurée par sa chute, ange victimé, s'impose néanmoins au terme de la nouvelle comme une allégorie de la poésie. Elle l'est d'une tout autre manière que la fée Euryale, dans "Le Renégat", ou la fée Tyro, dans «La Figurante». Certes, dans tous les récits de Banville, le lyrisme, indissociable du merveilleux, entretient une relation polémique avec le monde contemporain qui le condamne à une existence précaire et marginale. "Le sort de cette Psyché inconnue ne fut-il pas celui de la Poésie ignorante d'elle-même, toujours assassinée par les violences brutales de la vie ${ }^{42}$ ?", s'interroge le narrateur de La Vie d'une comédienne. L'allégorisation de Minette suggère aussi et surtout l'alliance du merveilleux et du naïf, elle présuppose une concordance harmonieuse entre le

39. Jeanne est publié en 1844 (Bruxelles, Haumann) et réédité avec une préface en I852 (Paris, Hetzel, Lecou).

40. Jeanne, Joué-Lès-Tours, Christian Pirot, 2006, p. 7.

4I. Ibid., p. 197.

42. Th. de Banville, La Vie d'une comédienne, ouvr. cité, p. 94. 
public populaire et la poésie, elle incite enfin à se demander quels rapports l'idéal entretient avec l'illusion.

Nodier, Sand, Nerval n'ont cessé de célébrer la poésie des chansons populaires. Jeanne d'Arc, selon Michelet, se serait nourrie des contes de fées et des vies de saints. Ces deux sources du merveilleux expliqueraient en partie les hallucinations de la jeune fille : elles tiennent, selon l'historien, d'une création poétique. La Pucelle, «à son insu, créait, pour ainsi parler, et réalisait ses propres idées, elle leur communiquait une partie du trésor de sa vie virginale, une splendide et toute puissante existence, à faire pâlir les réalités de ce monde. Si la poésie veut dire création, c'est là sans doute la poésie suprême ${ }^{43}$ ». Minette lectrice de contes de fées, à la différence de Jeanne d'Arc, n'actualise pas ses visions, ses rêves, dans une démarche héroïque, mais elle est l'ange de la croyance sans faille. Elle illustre hyperboliquement la naïveté du peuple qui "comprend intimement, profondément et de primesaut, la pensée du poète» et qui «seul est assez sincère, assez imaginatif, assez dénué d'idées fausses, contractées dans les études incomplètes et dans les fréquentations bourgeoises, en un mot assez ingénu, pour s'élever à la conception d'une féerie de Shakespeare ou d'une fable de La Fontaine ${ }^{44}{ }$. Si les titis du théâtre des Funambules voient «non pas à peu près, mais dans leur réalité absolue, les palais de rubis et de lapis ${ }^{45}$ " que des signes colorés sont censés évoquer, Minette projette, sur le monde noir qui l'accable, sa croyance dans la toute puissance des fées et regarde une féerie, ou plutôt la vit, en fonction de l'illusion qui informe sa perception du monde.

Cette notion d'illusion, dans l'univers de Banville, a une portée plurielle. Elle renvoie à la puissance trompeuse de l'amour qui transfigure l'être aimé. Cette capacité de voir en beau suscite une métamorphose merveilleuse dont une féerie écrite par Banville, parodie sérieuse du conte de Perrault, Riquet à la houppe, indique le ressort psychologique : "Pourtant je serai beau, si ma chère Princesse / Peut me voir ainsi, car l'Illusion sans cesse, / Nous transfigure, et sait d'un oiseau très banal, / Faire ce merle blanc qu'on nomme l'idéal ${ }^{46}$." L'illusion implique aussi une créance, un pacte mimétique : la représentation fictionnelle dit vrai. Les âmes simples le croient. Enfin, nous l'avons dit, elle est indissociable d'un défaut hermé-

43. J. Michelet, Jeanne d'Arc, Paris, Hachette, I853, cité d'après les Euvres complètes, Paris, Flammarion, 1978, t. III, p. 67.

44. Th. de Banville, Mes Souvenirs, Paris, Charpentier, I882, p. 22.

45. Ibid., p. 26.

46. Th. de Banville, Riquet à la houppe, Théâtre complet, ouvr. cité, t. III, p. I3O. 
neutique. Banville, dans La Vie d'une comédienne, joue avec la polysémie de l'illusion. Parce que ce récit n'est pas un conte, mais une nouvelle, publiée l'année même où Courbet expose ses toiles dans le Pavillon du réalisme, l'écrivain oppose au "principe de féerie" un principe de réalité, sans que soit dénoncé pour autant l'accord souterrain qui associe, dans un même paradigme, les mythes religieux, le merveilleux, la poésie. Ils ont pour essence commune d'être les fruits de l'imagination, voire de l'onirique. Avant même de découvrir les contes de fées, Minette compensait ses souffrances d'enfant battue en des songes tout illuminés d'images paradisiaques.

Peut-on délimiter en fonction de ces remarques les traits éventuels d'une littérature idéale susceptible de solliciter chez le créateur, le lecteur ou le spectateur, les facultés d'émerveillement et la puissance transfiguratrice de l'illusion? Pour Banville, les pantomimes données par Deburau au théâtre des Funambules en auraient esquissé les contours. Ce mime génial se serait suffisamment détaché de la réalité pour la figurer idéalement dans la dématérialisation même du jeu pantomimique. Il aurait pratiqué l'art de se "désaltérer d'un verre vide», de se "nourrir d'un festin absent ${ }^{47}$ ", suscitant ainsi une forme toute particulière de merveilleux. Les gestes de Deburau s'imposeraient presque comme de "purs signes». Parallèlement, le lyrisme, tel que le conçoit Banville, dans le conte féerique «Le Renégat», naît du silence. La fée Euryale "parlait plus souvent dans ce langage muet, et pourtant si réel, au moyen duquel les idées et les mots, sans être émis par une voix, arrivaient directement au cerveau et à l'entendement du poète ${ }^{48} »$. Banville qui, pour mieux exalter le merveilleux, dénonce, on l'a vu, la parodie blagueuse, l'académisme stérile, les féeries par trop spectaculaires, donne pour horizon ultime de l'idéalité, soit le silence pantomimique, soit le lyrisme naissant, qui dans son surgissement originel présuppose un dialogue silencieux avec soi.

Or, les contes féeriques ou les féeries de Banville sont bavards, à proportion de leur réflexivité, de leur ironie, de leur dimension polémique. Dénonçant la parodie, ils la pratiquent avec enjouement. C'est une tension d'un autre ordre que l'on aperçoit dans La Vie d'une comédienne. La poésie dont Minette est l'allégorie n’a aucune portée prophétique, aucune dimension heuristique ou herméneutique. Elle serait en quelque sorte un univers parallèle, un monde merveilleux qui pourrait s'auto-suffire si l'autotélisme rêvé, merveilleusement suspendu en l'air, à peine retenu par

47. Th. de Banville, Mes souvenirs, ouvr. cité, p. 23.

48. Th. de Banville, «Le Renégat», Contes féeriques, ouvr. cité, p. 202. 
les fils d'une féerie, n'était pas une chimère. Ange victimé, Minette est un martyr de l'illusion dont elle dit en même temps la splendeur. Enfin, si nous avons à plusieurs reprises mentionné la portée allégorique des Contes féeriques, ou celle de la longue nouvelle publiée en 1855, la relation ambivalente qu'entretiennent dans ces textes illusion et idéal témoigne que la clé allégorique proposée par l'écrivain à son lecteur est loin d'ouvrir toutes les portes. Le merveilleux banvillien ne se résorbe pas dans la morale qui est censée éclairer la portée de la fable. Situé en amont, mais aussi en aval, jamais actualisé, l'idéal figure un horizon lointain, fuyant et mélancolique. Le merveilleux, sous ses formes diverses, dit cet écartèlement. Il est, chez Banville, la manifestation éloquente d'une aspiration au silence, source et fin de la poésie. L'écrivain, il est vrai, dote parfois celle-ci d'un pouvoir magique : elle parviendrait à "exprimer l'inexprimable", à rendre "l'idéal palpable» en trouvant «des mots surhumains ${ }^{49}$ ». Nous verrons dans cette croyance une glorieuse illusion que La Vie d'une comédienne célèbre non sans la présenter comme telle.

49. Th. de Banville, Riquet à la houppe, ouvr. cité, t. III, p. II9. 